\title{
Trajectoires nobiliaires aux bordures et service du prince
}

Note de lecture de S. FOURCADE, D. LE PAGE et J. PAVIOT (dir.), La noblesse des marches, de Bourgogne et d'ailleurs au temps de Marguerite d'Autriche ( $\mathrm{xv}^{\mathrm{e}}-\mathrm{xvI} \mathrm{I}^{\mathrm{e}}$ siècles), éd. Annales de Bourgogne, tome 89, fascicules 3 et 4, Dijon, 2017, $265 \mathrm{p}$.

\section{Florentin Briffaz}

\section{(2) OpenEdition}

Journals

Édition électronique

URL : https://journals.openedition.org/cem/15347

DOI : $10.4000 /$ cem. 15347

ISSN : 1954-3093

Éditeur

Centre d'études médiévales Saint-Germain d'Auxerre

Référence électronique

Florentin Briffaz, «Trajectoires nobiliaires aux bordures et service du prince », Bulletin du centre d'études médiévales d'Auxerre | BUCEMA [En ligne], 22.1 | 2018, mis en ligne le 03 septembre 2018, consulté le 22 septembre 2022. URL : http://journals.openedition.org/cem/15347 ; DOI : https:// doi.org/10.4000/cem. 15347

Ce document a été généré automatiquement le 22 septembre 2022.

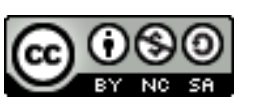

Creative Commons - Attribution - Pas d'Utilisation Commerciale - Partage dans les Mêmes Conditions 4.0 International - CC BY-NC-SA 4.0

https://creativecommons.org/licenses/by-nc-sa/4.0/ 


\section{Trajectoires nobiliaires aux bordures et service du prince}

Note de lecture de S. FOURCADE, D. LE PAGE et J. PAVIOT (dir.), La noblesse des marches, de Bourgogne et d'ailleurs au temps de Marguerite d'Autriche ( $\mathrm{XV}^{\mathrm{e}}-\mathrm{XVI}^{\mathrm{e}}$ siècles), éd. Annales de Bourgogne, tome 89, fascicules 3 et 4, Dijon, 2017, 265 p.

\section{Florentin Briffaz}

1 Parus en 2017 dans les Annales de Bourgogne, les actes du colloque international, organisé par Sarah Fourcade, Dominique Le Page et Jacques Paviot, les 14 et 15 septembre 2016 à Brou, monastère fondé par Marguerite d'Autriche, la jeune veuve du duc de Savoie Philibert le Beau, constituent un jalon important dans l'historiographie de la noblesse. L'ouvrage apporte beaucoup de matière à réflexion, tant sur le plan des études locales et régionales identifications d'individus, trajectoires de lignages, aperçu des constructions politiques et territoriales princières - que sur le plan de considérations plus générales autour de la question nobiliaire.

2 La présente publication se veut, en effet, précisément structurée autour de la

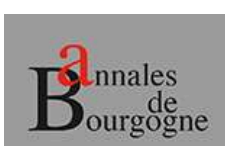

TOME 89 - FASCICULES 3 et 4 - 2017

La noblesse des marches, de Bourgogne et d'ailleurs au temps de Marguerite d'Autriche (XV'-XVI' siècle)

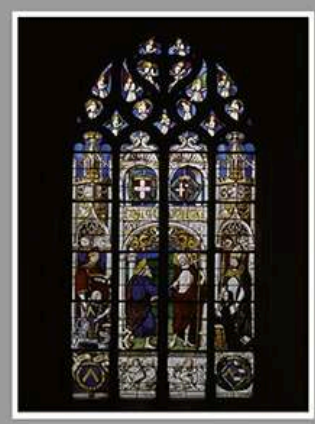

DID thématique des marches et ce, dans un espace géographique et politique bien précis, qui n'est pas sans rappeler furieusement une partie de la dorsale lotharingienne ${ }^{1}$. L'ouvrage tient ainsi une vraie cohérence, depuis l'avant-propos de Philippe Contamine, l'introduction de Jacques Paviot et tout 
au long des treize contributions, qui sont autant d'études de cas menées par chaque spécialiste à la lumière d'une lecture attentive des sources. Contrairement à ce qui est annoncé dans le titre du volume, le travail ne se cantonne pas à la période charnière des $\mathrm{XV}^{\mathrm{e}}-\mathrm{XVI}^{\mathrm{e}}$ siècles, mais déborde nécessairement en amont et en aval.

D'emblée, la parole est donnée à Philippe Contamine ${ }^{2}$ à qui l'on doit une synthèse sur la noblesse dans le royaume de France ${ }^{3}$. Ajoutons à ce tableau la toute récente biographie qu'il a consacrée à Charles VII ${ }^{4}$, roi pour lequel la question des marches est d'une certaine prégnance, notamment si l'on songe à la question de la reconquête du royaume. P. Contamine historicise le concept et la réalité - quand bien même mouvante - des marches, tout en proposant à grands traits une mise en perspective étymologique stimulante de ce terme.

4 Une première remarque s'impose. Certes, la thématique nobiliaire a été l'objet ces dernières années de plusieurs travaux, au premier rang desquels figurent ceux de Marie-Thérèse Caron, de Guido Castelnuovo et de Martin Aurell pour la période médiévale ${ }^{5}$, de Michel Nassiet pour la charnière entre le $\mathrm{xv}^{\mathrm{e}}$ et le $\mathrm{xvI}^{\mathrm{e}}$ siècle ${ }^{6}$ et, en particulier, de Laurent Bourquin, porte-étendard du concept de "noblesse seconde » forgé par Jean-Marie Constant, pour la première modernité ${ }^{7}$. Jacques Paviot brosse ainsi dans son propos liminaire le tableau de cette historiographie ${ }^{8}$ et montre, à juste titre, que cette dernière peut parfois relever d'une tradition fort ancienne. On songe ici aux travaux incontournables du grand érudit du XVII e siècle, Samuel Guichenon, portant sur la Bresse et le Bugey. Mais si la noblesse fut très tôt un objet d'étude prisé, la question spécifique des marches, notamment du côté de la Bourgogne et des terres voisines, demeura largement laissée de côté; ce à quoi promettait de remédier le colloque de Brou.

5 Pour autant, il faudrait insister sur le fait que la thématique de la noblesse des marches est un champ de recherches qui a été abordé sans doute depuis plus longtemps et avec plus de force par l'historiographie bretonne. D'ailleurs, quel nom vient spontanément à l'esprit? De toute évidence celui, illustre, de Roland, «comte des marches de Bretagne ", véritable figure tutélaire aux frontières du réel et de l'imaginaire. De fait, les travaux sur les marches bretonnes ne manquent pas ; citons en premier lieu ceux de René Cintré et de Laurence Moal ${ }^{9}$. Cela s'inscrit peut-être dans une logique toute particulière puisque les Marches de Bretagne désignent un espace - fluctuant et belligène s'il en est - hérissé de forteresses. C'est dans cette veine de réévaluation historiographique que s'inscrivait l'exposition « Les Marches de Bretagne, les frontières de l'histoire ", tenue en 2009-2010 au château de Châteaubriant, une des deux principales « entrées et yssues de ce pays et duché de Bretagne » avec Clisson.

6 Après cette brève mise en contexte, on le voit donc, la noblesse des marches est une question d'actualité en Bretagne, alors que les recherches portant spécifiquement sur cet aspect dans l'espace burgundo-savoyard sont moins nombreuses, même si plusieurs études parcellaires existent parfois depuis longtemps ${ }^{10}$. Ce faisant, on ne peut que vanter les mérites des actes du colloque de Brou, tout en plaidant pour un comparatisme renforcé avec l'État breton.

7 Les marches posent le problème épineux de la frontière, terme que certains historiens renâclent à employer pour le Moyen Âge. Il s'agit d'un espace mouvant que les potentats tentent de contrôler par la construction de places fortes et de forteresses. Cette question est d'une réelle acuité pour la Savoie et il faudrait ici davantage mettre l'accent sur les travaux d'Alain Kersuzan sur la Bresse et le Bugey du temps de la guerre 
delphino-savoyarde ${ }^{11}$, travaux qui ont montré combien nombre de châteaux ou de bâties, plus ou moins éphémères - à l'instar de la bâtie de Luisandre ${ }^{12}$-, étaient nés au moment de "la guerre de septante" et avaient hérissé ces marges montagnardes contestées. Un autre témoignage est fourni par la toponymie locale avec les Marches, commune viticole actuelle de la Combe de Savoie, qui trouve son origine dans la création en 1301 d'une villeneuve en interface avec le Dauphiné et dotée de franchises par le comte de Savoie en 1304. Une nouvelle fois, le caractère belliqueux ressort avec évidence dans cette région à forte valeur ajoutée qu'est la "Marche de Savoie ", pour reprendre la formule de Pierre Brugnon, dont la contribution au présent volume s'attache entre autres à démêler l'écheveau de lignages aristocratiques implantés dans cet espace et à suivre les trajectoires de ces noblesses ligueuses ${ }^{13}$.

8 De fait, l'un des enjeux et des apports du livre est, au moyen d'études de cas rondement menées, l'identification d'une série d'individus et de lignages œuvrant aux marches d'espaces politiques.

9 Mais ceci est à double tranchant et les lignages jouent un jeu dangereux ; d'un côté, on peut aisément imaginer les fruits d'un partage de services, au profit d'intérêts personnels ou familiaux, notamment entre le Royaume et l'Empire ; de l'autre côté, les accusations de trahison peuvent arriver plus vite que prévu, quand bien même la clause de ligesse d'hommage aurait été érigée en barrière.

C'est toute cette ambiguïté qui se retrouve au cœur des investigations du volume et qui constituait déjà en projet le fil d'Ariane du colloque, ce qu'explique en avant-propos $\mathrm{P}$. Contamine :

Clairement, le dessein du futur colloque visait à évoquer, sous un angle principalement politique, des individus ou des lignages nobles détenteurs de terres et de châteaux situés au contact de deux dominations, ce qui était peut-être pour eux un handicap mais aussi un bénéfice: ne pouvaient-ils pas en profiter pour se faire payer leur fidélité, en faisant monter les enchères, mais ne risquaient-ils pas en revanche d'être accusés de traitrise par l'un ou l'autre de leurs suzerains ou souverains, voire par les deux? (p. 8).

11 À cette question, les contributions répondent par des exemples précis, parfois en écho.

12 Ainsi, la famille bressane des La Baume, avec comme figure de proue Galois de La Baume, grand maître des arbalétriers de France, est évoquée par J. Paviot puis par P. Brugnon dans le sens d'une illustration du dédoublement des fidélités. La Bresse fait, en effet, largement partie des États nord-occidentaux de Savoie. Elle constitue cependant une zone de confins, qui aimante les influences variées de voisins plus ou moins puissants. Celles-ci sont culturelles - le temps de Marguerite d'Autriche est l'occasion d'un mécénat à l'égard d'artistes venant, notamment, des Pays-Bas bourguignons -, mais également politiques - on songera à l'ingérence du duc de Bourgogne en Savoie du temps de la minorité d'Amédée VIII, fait marquant qu'avait déjà étudié, voilà plus de cent ans, Francesco Cognasso ${ }^{14}$. Les lignages peuvent tirer profit de cette situation et l'exemple de Galois de La Baume est l'acmé d'un processus conduisant à mettre sur le devant de la scène certains individus, dont la renommée peut retomber ou non sur la famille dont ils sont issus.

La situation de marches n'en constitue pas moins assez souvent un dilemme pour toutes ces constructions lignagères. La noblesse établie en ces lieux peut parfois être fort turbulente. C'est l'occasion de compétitions en matière de juridictions territoriales ou de préséance sociale et politique. Il en est ainsi du conflit étudié par P. Brugnon 
entre Montmayeur et Fésigny, qui rappelle l'opposition entre noblesse d'épée et noblesse de robe. De surcroit, une telle distinction est à nuancer. Mais la typologie même de la noblesse et la fluctuation de ses contours constituent une question fort préoccupante, y compris dans ces zones de marches et c'est un versant qui a peut-être été moins exploré lors des actes de ce colloque.

L'espace considéré peut être alors travaillé par des forces centrifuges et concurrentes. Tout ceci pose inévitablement la question des formes d'encadrement de la noblesse de ces régions. Les ordres de chevalerie apparaissent comme l'un des moyens privilégiés de fidélisation de ces lignages, parfois peu enclins à honorer une vassalité, sinon restrictive, du moins préférentielle. Thalia Brero évoque cette problématique dans son article portant sur l'ordre savoyard de l'Annonciade ${ }^{15}$, successeur de l'ordre du Collier par la refonte de 1518 voulue par le duc Charles II. L'auteur s'appuie, en particulier, sur les travaux fondamentaux de Laurent Ripart pour la Savoie et ceux, plus généraux, de D'Arcy Boulton ${ }^{16}$. En outre, elle souligne le poids des événements; la réactivation de l'ordre en 1518 est à certains égards une tentative de réponse aux défis du temps, à commencer par les menaces liées aux guerres d'Italie. Bien que la nuance soit de mise, la noblesse des marches méridionales de l'État savoyard semble alors favorisée par les promotions ducales (p. 72). Il en va de la survie de l'État alpin face aux appétits de son voisin français.

Tout tourne une nouvelle fois autour du service du seigneur éminent, qui est le fil rouge des différentes études rassemblées ici. Et la promotion au rang de chevalier est un instrument privilégié de cette politique, entre récompense individuelle et structuration de la société nobiliaire autour de valeurs communes, à commencer par l'obéissance au prince, sorte de primus inter pares, et ce, même si, comme le fait remarquer T. Brero pour la Savoie, des solidarités horizontales ont pu prévaloir lors du premier âge de l'ordre.

16 Mais la question du service chez les nobles des marches entraîne parfois des divisions intra-lignagères; le choix du prince à servir pouvant être la pomme de discorde et la matière à distinction dans les fratries aristocratiques. Vianney Muller met en évidence ces problèmes de positionnement à la lumière de l'étude fine des NeufchâtelBourgogne ${ }^{17}$, famille à laquelle il a consacré sa thèse ${ }^{18}$. Les frères ne suivent pas nécessairement les mêmes fidélités et ne se sentent parfois pas obligés de se porter une assistance mutuelle en vertu d'une solidarité lignagère. V. Muller, en comparant les trajectoires des deux frères Thiébaut IX (1414-1469) et Jean II (1415-1489), rapporte ainsi, fort à propos, le cri du cœur de ce dernier : «C'est aussi chose bien clere que ung frere ne doit estre contraint a faire guerre pour l'autre, se ce n'est de son bon grey et plaisir » (p. 185).

17 La contribution de Florence Beaume, consacrée au parcours comparé des deux frères Gorrevod, Laurent (v. 1470-1529) et Louis (v. 1473-1536), à l'aune de leur proximité avec Marguerite d'Autriche, entre également en résonance ${ }^{19}$. L'aîné finit au sommet de sa gloire, dans la faveur intacte de l'empereur, à l'inverse du cadet qui décède dans "l'obscurité et l'humilité» (p.28). F. Beaume retrace l'ascension de Laurent de Gorrevod. L'obtention de charges prestigieuses et stratégiques permet d'accroître la renommée du serviteur et la richesse de l'homme ${ }^{20}$. Il en vient à occuper de très hautes fonctions à la cour de Charles Quint. Son élévation le 26 octobre 1516 au rang de chevalier de l'ordre de la Toison d'or est un tournant (p. 21). Elle témoigne du degré de confiance accordé par l'empereur. 
18 En outre, le problème des fidélités se pose avec une acuité toute particulière lors de la mort du seigneur éminent ou du chef de la souveraineté. C'est l'un des grands apports du présent volume que de mettre en perspective certains moments de rupture et de crises, comme la mort de Charles le Téméraire à la bataille de Nancy en 1477. Ainsi, Hans Cools, dont la contribution porte sur la figure de René de Chalon ${ }^{21}$, a fait une étude fondamentale sur les trajectoires nobiliaires de 1475 à 1530, c'est-à-dire, d'une certaine façon, en prenant pour point de bascule la mort du prince en $1477^{22}$. Les noblesses doivent faire face à une recomposition, à un repositionnement, entre stratégie politique et parfois survie familiale. C'est tout l'enjeu d'étude de plusieurs articles. V. Muller montre le vide laissé par la mort du duc et le défi que celle-ci occasionne. Les branches du lignage des Neufchâtel-Bourgogne ne sont cependant pas dans la même situation et le pouvoir d'attraction du roi Louis XI est un facteur décisif de recompositions passant par des ralliements plus ou moins précoces, eux-mêmes inhérents à « la capacité de négociation » (V. Muller, p. 198).

L'événement nancéen laisse d'autant plus de traces qu'il est une défaite cuisante. Ce peut être une mémoire tragique. Ainsi, Sarah Fourcade étudie avec précision le destin des Du Chastel de la Howarderie ${ }^{23}$ au miroir d'un «livre de famille» manuscrit ${ }^{24}$, élaboré dès les années 1510 par Simon du Chastel de la Howarderie. Cette forme d'épopée familiale, couplée à des leçons ${ }^{25}$ dispensées à son fils, est teintée de nostalgie. Comme le montre très bien $\mathrm{S}$. Fourcade chez Simon du Chastel, il existe un avant et un après 1477. Le seigneur éprouve un fort sentiment de déchéance.

Cette contribution s'inscrit ainsi bien dans l'approche synchronique - et non simplement diachronique -, qui est un des points forts du colloque. De la même façon, Céline Berry s'attelle à l'étude du lignage des Luxembourg après $1475^{26}$. C'est assurément un moment de rupture dans la mesure où Louis de Luxembourg, connétable de Saint-Pol, est exécuté sur ordre de Louis XI pour crime de lèse-majesté, à la suite d'un célèbre procès devant le Parlement. Sa chute brutale rebat les cartes; la tradition de service multiple n'étant plus guère possible, il faut choisir son camp. Là où la branche aînée opte pour la France, d'autres membres du lignage demeurent attachés au service du duc de Bourgogne. 1475 est à tous égards l'« année terrible » pour la Maison, « un tournant décisif dans l'histoire des Luxembourg » (C. Berry, p. 224) : le connétable est condamné à mort, ses biens sont confisqués et son frère Jacques et son fils Antoine sont retenus dans les geôles de Louis XI.

21 Dans la même veine, la contribution d'Anne-Valérie Solignat s'intéresse à un autre épisode célèbre: la trahison du connétable de Bourbon en $1523^{27}$. Elle en étudie notamment les conséquences chez la noblesse auvergnate et inscrit son propos dans une réflexion au long cours sur les forces et les faiblesses des ducs de Bourbon et de l'État bourbonnais aux Xve et $\mathrm{xVI}^{\mathrm{e}}$ siècles, prolongeant, sur le terrain d'investigation du service princier et du monde des officiers, les travaux fondamentaux d'olivier Mattéoni ${ }^{28}$. La trahison de Charles de Bourbon, «le dernier des grands féodaux » (A.V. Solignat, p. 236), délie la fidélité que ces lignages - déjà entrés pour certains dans la mouvance du roi de France ou d'autres potentats compétiteurs comme le prince d'Armagnac - lui devaient. Mouvance, turbulence, ambiguïté, duplicité... Tels sont les termes qui peuvent émailler la vision que l'on a des noblesses implantées dans les marches.

Se forge alors une légende noire qui corsète le destin de ces personnages ondoyants aux allures de baroudeurs, et ce, parfois jusque dans l'historiographie la plus récente. Il en 
est ainsi du sire de Commercy, Robert de Sarrebrück (1400-1462), dont Valérie Toureille retrace avec précision le parcours aux confins de la France, de la Lorraine et de la Bourgogne ${ }^{29}$. Le personnage évolue dans un contexte troublé, en pleine guerre de Cent Ans. La mouvance même de la frontière et la concurrence, plus ou moins aiguë entre les différents pouvoirs qui s'organisent autour d'elle, peuvent être pleinement intériorisées par un «seigneur bordurier » comme Robert de Sarrebruck (V. Toureille, p. 162). L'auteur insiste sur le fait que la marche est un espace vécu et qu'il convient de porter le regard sur les individus et les lignages qui se l'approprient et qui la façonnent à différents niveaux. Plus encore, l'étude, à nouveaux frais, de la carrière de Robert de Sarrebrück, menée par V. Toureille, est à rapprocher de son autre contribution au très beau colloque de Berbiguières sur les routiers et les mercenaires pendant la guerre de Cent $\mathrm{Ans}^{30}$. Il faudrait, en effet, véritablement mettre l'accent sur la parenté entre les deux approches. La question des routiers et des mercenaires, par-delà la complexité terminologique et la polysémie de ces notions, est en réalité assez souvent une question de marches. Des nobles, issus de lignages plus ou moins grands, mais établis dans des zones de confins, peuvent mener de fructueuses carrières de routiers et devenir des soutiens privilégiés en raison de leurs possessions ou de leurs prises stratégiques.

La démarche de $\mathrm{V}$. Toureille est à mettre en parallèle avec celle opérée par Dominique Le Page pour Jean IV de Chalon (1443-1502), grande figure complexe issue d'un très haut lignage des deux Bourgogne, ramifié à l'envi ${ }^{31}$. Parce qu'il passa du service de Louis XI à celui de Marie de Bourgogne, tout en œuvrant sur le terrain breton, Jean de Chalon pâtit très tôt d'une mauvaise réputation, celle d'un grand noble des marches à la fidélité douteuse. D. Le Page propose une lecture critique de cette légende noire, retraçant patiemment les grands jalons de cette historiographie à charge, et ce, depuis les chroniqueurs bourguignons médiévaux jusqu'aux travaux les plus récents des historiens.

Ainsi, la contribution de D. Le Page est symptomatique de l'ambition du présent ouvrage de dialoguer avec l'actualité de la recherche en histoire. L'auteur évoque en préambule le beau livre remarqué d'Élodie Lecuppre-Desjardin sur «le royaume inachevé des ducs de Bourgogne ${ }^{32}$ et critique le fait que celle-ci fasse de Jean de Chalon «le modèle des transfuges" (p.125). D. Le Page insiste au contraire sur l'amplitude d'action de Jean de Chalon et sur la nécessité de réévaluer son investissement sur la scène bretonne.

C'est bel et bien un enjeu méthodologique fondamental qui ressort de cette étude. Il convient effectivement de suivre attentivement les trajectoires des individus et des lignages. La noblesse des marches est fortement marquée par la question des mobilités et des transferts de fidélités. F. Beaume met en exergue les difficultés méthodologiques et pratiques que rencontre le chercheur pour pister ces individus et pour rassembler la documentation disponible. Il en est ainsi pour saisir l'action de Laurent de Gorrevod en Amérique dans le cadre de la traite transatlantique.

Les actes du colloque sont également l'occasion de mises au point. Par exemple, Cédric Mottier, au début de son article sur les Coucy-Châteauvieux ${ }^{33}$, propose une relecture de Guichenon, tout en complétant les travaux de ce dernier par de nouveaux documents originaux extraits des différents fonds d'archives. De même, Pierre-Gilles Girault démonte méthodiquement tous les mythes colportés autour des Carondelet ${ }^{34}$, à commencer par les généalogies douteuses établies au cours des siècles. Il dévoile ainsi au lecteur les doutes et les avancées du chercheur. Ce faisant, il en vient à démontrer 
que les lettres de reconnaissance du duc de Bourgogne, Philippe le Bon, prétendument datées de 1430 et produites comme preuves de noblesse par la famille plus tard, sont en réalité une forgerie de cette dernière $\mathrm{du} \mathrm{xVIII}^{\mathrm{e}}$ siècle.

En outre, la publication est l'occasion de discussions, de réflexions autour de concepts liés à la noblesse. Ainsi, dans son étude consacrée aux rapports entre la France et la noblesse bourguignonne au lendemain de la victoire d'Henri IV à la bataille de Fontaine-Française en 1595, Jérôme Loiseau s'intéresse au concept de «noblesse seconde » forgé par Jean-Marie Constant en 1989 et utilisé, notamment, par Laurent Bourquin. Il en retrace la genèse, avant de tenter d'adapter la notion au cas bourguignon $^{35}$. En somme, la noblesse bourguignonne devient un révélateur, un test pour juger de la pertinence d'un concept, dont J. Loiseau met en évidence les forces et les faiblesses. L'auteur retrace ainsi les débats autour du sujet et les critiques formulées dès les prodromes par certains historiens comme Robert Descimon. J. Loiseau poursuit l'investigation et se demande si la notion ne serait pas plus acceptable dans le cas des marches, des provinces frontalières. Il s'interroge alors sur le cas de Charles de Damas, sieur de Thianges, un de ces «nobles dits seconds» (p. 84), promus par la monarchie française, en l'occurrence ici par Richelieu, qui entend bâtir une véritable clientèle. J. Loiseau montre toute la complexité de l'usage du concept de noblesse seconde; si Thianges est un relais du pouvoir royal en Bourgogne - il est même lieutenant du roi en Bresse et en Charolais - face aux agissements et aux velléités de contestation d'un Condé ou d'un Gaston d'Orléans, il n'efface pas pour autant les autres parties en présence. L'autorité royale laisse ainsi perdurer à côté le système concurrentiel des grands et des princes du sang.

Par conséquent, on aura mesuré la richesse du colloque et la variété des exemples mobilisés au miroir spécialement de la question du service. Le versant artistique n'est pourtant pas oublié. L'étude de P.-G. Girault porte ainsi sur le mécénat exercé par les Carondelet. L'auteur montre que la commande artistique est un moyen fort prisé par ce lignage pour servir une ascension politique et pour gagner une certaine prééminence sociale. C'est particulièrement vrai pour Ferry (1473-1528), qui, à l'occasion d'un séjour à Rome en tant que procureur impérial auprès du pape, passe commande auprès d'artistes de grand renom, comme Sebastiano del Piombo ou encore Fra Bartolomeo.

En définitive, il ressort du volume d'actes une très grande richesse dans la mobilisation et l'analyse de sources de première main. Il faut ici saluer la présence de nombreuses généalogies, qui permettent de bien visualiser les lignages étudiés lors de chaque contribution et qui apportent une grande clarté au discours. Certains articles s'accompagnent également de cartes qui servent fort à propos la démonstration. Elles mettent en évidence les phénomènes de patrimonialisation, autre vecteur possible d'ascension politique et sociale. Les images d'objets ou de constructions monumentales, en annexes des contributions de F. Beaume et de P.-G. Girault, rappellent la place importante dévolue à l'art et aux commandes artistiques dans les constructions nobiliaires. De plus, dans le cas de la contribution de T. Brero, la liste des chevaliers de l'Annonciade promus par Charles II de Savoie, assortie d'un petit régeste rappelant les grandes lignes du parcours de chaque membre de cette societas, permet de bien identifier les lignages nobles des marches de l'État savoyard suivant une approche prosopographique.

31 Le présent ouvrage constitue un jalon important dans l'historiographie nobiliaire avec cette réflexion fructueuse sur la question complexe des marches. La matière déployée 
ici est abondante et permet des études à toutes les échelles et avec différents degrés de comparatisme. On regrettera cependant l'absence d'un index de lieux et de personnes. Les actes du colloque de Brou susciteront, à n'en point douter, des vocations de recherches. Dans son sillage, on peut déjà souligner la tenue en septembre 2017 d'un colloque portant sur la noblesse des Bourgogne du $\mathrm{XIV}^{\mathrm{e}}$ au $\mathrm{XVI}^{\mathrm{e}}$ siècle autour de l'exemple développé de la famille de Chalon, cas somme toute à la fois typique et atypique de la noblesse des marches et des défis auxquels elle est confrontée ${ }^{36}$.

Reçu : 27 janvier 2018 - Accepté : 6 juin 2018

\section{NOTES}

1. La thématique «lotharingienne " est en plein renouvellement. On peut citer le projet ANR LODOCAT (Chrétientés lotharingiennes - Dorsale catholique, IX $\mathrm{IX}^{\mathrm{e}}$-XVIII ${ }^{\mathrm{e}}$ siècle) coordonné par Christine Barralis, de l'université de Lorraine. Il faut mentionner également tout particulièrement la thèse de Tristan Martine, sous la co-direction de Geneviève Bührer-Thierry et de Gérard Giuliato, intitulée « Ancrage spatial et polarisation des pouvoirs de l'aristocratie laïque en Lotharingie méridionale (fin $\mathrm{IX}^{\mathrm{e}}-\mathrm{mi} \mathrm{XI}^{\mathrm{e}}$ siècle) » et soutenue le 25 novembre 2017 à l'université Paris-Est Marne-la-Vallée. Cet espace, entendu dans son sens large, y compris pour des époques postérieures à l'ère de «l'axe lotharingien» défini par Michel Parisse, fait ainsi l'objet de plusieurs recherches mettant en évidence son dynamisme et ses particularismes liés justement à sa situation de marches.

2. P. ContAmine, « Avant-propos », p. 7-9.

3. P. ContAmine, La noblesse au royaume de France, de Philippe le Bel à Louis XII, Paris, 1997.

4. P. Contamine, Charles VII. Une vie, une politique, Paris, 2017.

5. Ce sont des travaux issus de traditions historiographiques parfois différentes, mais qui se fondent sur l'étude d'espaces régionaux et princiers bien délimités. Voir, entre autres, M.T. CARON, La noblesse dans le duché de Bourgogne, 1315-1477, Lille, 1987 ; G. CASTELnUovo, Ufficiali e gentiluomini. La società politica sabauda nel tardo medioevo, Milan, 1994 ; ID., Seigneurs et lignages dans le Pays de Vaud. Du royaume de Bourgogne à l'arrivée des Savoie, Lausanne, 1994 ; ID., " Nobles des champs ou nobles de cour? Princes et noblesse dans les chroniques savoyardes du $\mathrm{Xv}^{\mathrm{e}}$ siècle », in M. GENTILE et P. SAVY (éd.), Noblesse et États princiers en Italie et en France au XVe siècle, Rome, 2009, p. 191-208. Enfin, les très nombreuses publications de Martin Aurell, passées sous silence dans les actes du colloque de Brou, mériteraient ici d'être signalées, notamment : M. AURELL, Une famille de la noblesse provençale au Moyen Âge : les Porcelet, Avignon, 1986 ; ID., « Le lignage aristocratique en Provence au XI ${ }^{\mathrm{e}}$ siècle ", Annales du Midi, 98 (1986), p. 149-163; ID. (éd.), Noblesses de l'espace Plantagenêt (1154-1224), Poitiers, 2001. Les enquêtes de Martin Aurell portent également plus spécifiquement sur le thème et l'espace des marches, avec l'exemple de la marche d'Espagne et du comté de Barcelone; cf. ainsi : ID., Les noces du comte : mariage et pouvoir en Catalogne (785-1213), Paris, 1995 ; ID., « Pouvoir et parenté des comtes de la marche hispanique (801-911) », in R. LE JAN (dir.), La royauté et les élites dans l'Europe carolingienne (du début du IX aux environs de 920), Lille, 1998, p. 467-486.

6. Cf. spécialement M. NASSIET, Parenté, noblesse et États dynastiques, $\mathrm{XV}^{e}-\mathrm{XVI}{ }^{e}$ siècles, Paris, 2000. 
7. L. BourQuin, Noblesse seconde et pouvoir en Champagne aux XVI et XVII siècles, Paris, 1994 ; ID., Les nobles, la ville et le roi. L'autorité nobiliaire en Anjou pendant les guerres de Religion (1560-1598), Paris, 2001 ; ID., La noblesse dans la France moderne, XVI ${ }^{e}$-XVIII ${ }^{e}$ siècle, Paris, 2002 ; J.-M. CONSTANT, «Un groupe socio-politique stratégique dans la France de la première moitié du XVII siècle: la noblesse seconde ", in P. ConTAMINE (éd.), L'État et les aristocraties (France, Angleterre, Écosse), XII XVII ${ }^{e}$ siècle, Paris, 1989, p. 279-304.

8. J. PAVIOT, «Introduction ", p. 11-17. En outre, les propres travaux de J. Paviot entrent ici en résonance tant sur le plan de l'histoire des pratiques sociales que sur le plan de l'aire géographique. Ses recherches sont largement consacrées à la noblesse, à l'espace bourguignon et aux heurs et malheurs de l'esprit de croisade à la fin du Moyen Âge. Or, les rapports entre la noblesse et la croisade s'inscrivent dans la thématique des marches, puisque ces entreprises aristocratiques visent souvent des territoires de confins. Cf. ainsi J. PAVIOT, Les ducs de Bourgogne, la croisade et l'Orient (fin XIV siècle-XV $V^{e}$ siècle), Paris, 2003; ID., Projets de croisade (v. 1290-v. 1330), Paris, 2008 ; ID. (dir.), Les projets de croisade. Géostratégie et diplomatique européenne du XIV au XVII siècle, Toulouse, 2014. Pour l'espace plus précis des pays de l'Ain, voir les recherches du même auteur sur la famille de La Palud, véritable laboratoire d'étude de la question des marches et de l'ambiguïté des fidélités. Cf. notamment: ID., «François de La Palud, seigneur de Varambon, un encombrant seigneur du XV siècle ", in P. GILLI et J. PAVIOT (dir.), Hommes, cultures et sociétés à la fin du Moyen Âge. Liber discipulorum en l'honneur de Philippe Contamine, Paris, 2012, p. 257-292.

9. R. CINTRÉ, La frontière franco-bretonne au Moyen Âge. Économie, mentalités, guerre et société en pays de marches, $\mathrm{VI}^{e}-\mathrm{XVI}^{e}$ siècle, thèse de doctorat en histoire, sous la direction de Jean-Pierre Leguay, université de Rouen, 1989 ; ID., Les marches de Bretagne au Moyen Âge : économie, guerre et société en pays de frontière (XIV -XV $V^{e}$ siècles), Pornichet, 1992; ID., "Entre Bretagne et Normandie, les problèmes de la frontière franco-bretonne $a u X^{e}$ siècle ", in B. MERDRIGNAC et J. QUAGHEBEUR (dir.), Bretons et Normands au Moyen Âge. Rivalités, malentendus, convergences, Rennes, 2008, p. 301-314 ; L. MOAL, «De la seigneurie à l'État princier: les frontières en mouvement ( $\mathrm{xl}^{\mathrm{e}}-\mathrm{XV}^{\mathrm{e}}$ siècle) ", in M. CATALA, J.-C. MEURET et D. LE PAGE (dir.), Frontières oubliées, frontières retrouvées. Marches et limites anciennes en France et en Europe, Rennes, 2011, p. 209-220. Pour une très belle mise en perspective des enjeux de recherches autour de la marche et de la notion même de frontière, à l'aune d'un comparatisme renforcé entre principautés médiévales, cf. l'article plus récent de Laurence Moal : $E A D$., « Dans le royaume ou en marge ? Les frontières des principautés (XIII $-\mathrm{XV}^{\mathrm{e}}$ siècle) ", Annales de Bretagne et des Pays de l'Ouest, 121/2 (2014), p. 47-81.

10. B. DемотZ, «La frontière au Moyen Âge d'après l'exemple du comté de Savoie (début XIII début $\mathrm{XV}^{\mathrm{e}}$ siècles) ", in Les principautés au Moyen Âge. Actes $d u 4^{e}$ congrès de la Société des historiens médiévistes de l'enseignement supérieur public, Bordeaux, 1973, Bordeaux, 1979, p. 95-116.

11. A. KERSUZAN, Défendre la Bresse et le Bugey. Les châteaux savoyards dans la guerre contre le Dauphiné (1282-1355), Lyon, 2005 ; ID., Châteaux et fortifications au Moyen Âge dans l'Ain des montagnes, Bourgen-Bresse, 2015.

12. A. KERSUZAN, La bâtie de Luisandre. Histoire et archéologie d'une fortification savoyarde de frontière au XIV siècle, Chambéry, 2010.

13. P. B RUGNON, «Nobles des marches et nobles des princes dans l'ancien royaume de Bourgogne : le cas des noblesses savoyardes ( $\mathrm{XIV}^{\mathrm{e}}-\mathrm{XV}^{\mathrm{e}}$ siècle) », p. 107-124.

14. F. Cognasso, "L'influsso francese nello Stato sabaudo durante la minorità di Amedeo VIII Note e documenti inediti », Mélanges d'archéologie et d'histoire, 35 (1915), p. 257-326.

15. T. BRERO, «La loyauté contre un collier. L'ordre de chevalerie savoyard, instrument de fidélisation de l'aristocratie frontalière? », p. 57-75.

16. L. RIPART, « Du Cygne noir au Collier de Savoie : genèse d'un ordre monarchique de chevalerie (milieu XIve-début XV $\mathrm{XV}^{\mathrm{e}}$ siècle) », in L. GENTILE et P. BIANCHI (éd.), L'affermarsi della corte sabauda. 
Dinastie, poteri, "élites" in Piemonte e Savoia fra tardo medioevo e prima età moderna, Turin, 2006, p. 93-113; D. Boulton, The Knights of the Crown. The Monarchical Orders of Knighthood in Later Medieval Europe, 1325-1520, Woodbridge, 2000.

17. V. MULLER, «Entre fidélité et pragmatisme : le lignage de Neufchâtel-Bourgogne au cœur de la tourmente », p. 177-199.

18. Le patrimoine fortifié du lignage de Neufchâtel-Bourgogne (XIII -XVI ${ }^{e}$ siècle), thèse de doctorat en histoire, sous la direction de Gérard Giuliato, soutenue le 13 juin 2015 à l'université de Lorraine.

19. F. BEAUME, « Les Gorrevod, de la Bresse à l'Empire », p. 19-32.

20. Il commence à agréger les charges dans sa province natale, devenant ainsi, dès 1504, gouverneur de Bresse.

21. H. Cools, « Les Orange-Nassau comme princes bourguignons : l'accession de René de Chalon à la principauté d'Orange et aux territoires francs-comtois », p. 149-159.

22. H. Cools, Mannen met macht. Edellieden en de Moderne Staat in de Bourgondisch-Habsburgse landen (1475-1530), Zutphen, 2001.

23. S. F OURCADE, "Servir après 1477 : les du Chastel de la Howarderie entre Bourgogne et France ", p. 201-215.

24. PARIS, Bibliothèque nationale de France, ms. français 11602.

25. Voir l'«Exhortation" adressée à son fils Jacques, sorte de leçon édifiante et pessimiste, prenant appui sur son expérience des malheurs de la vie aristocratique (éd. S. Fourcade, p. 213-214).

26. C. BERRY, "Après la mort du connétable de Saint-Pol. Les Luxembourg entre France et Bourgogne ", p. 217-233. C. Berry a précisément consacré sa thèse à cette famille : Les LuxembourgLigny, un grand lignage noble de la fin du Moyen Âge, thèse en histoire, sous la direction de Jacques Paviot, soutenue le 12 décembre 2011 à l'université Paris-Est-Créteil.

27. A.-V. Solignat, «La noblesse d'Auvergne et la trahison du connétable de Bourbon (1523) », p. 235-251.

28. O. MATTÉonI, Servir le prince. Les officiers des ducs de Bourbon à la fin du Moyen Âge (1356-1523), Paris, 1998.

29. V. TOUREILLE, «Entre France, Lorraine et Bourgogne : Robert de Sarrebruck, un seigneur de guerre aux frontières (1400-1462)», p. 161-175.

30. V. TOUReILlE, «Robert de Sarrebrück, un routier au service de Charles VII », in G. P ÉPIN, F. LAINÉ et F. B Outoulle (dir.), Routiers et mercenaires pendant la guerre de Cent Ans. Hommage à Jonathan Sumption, Bordeaux, 2016, p. 179-188.

31. D. LE PAGE, « Jean IV de Chalon, prince d'Orange entre Bourgogne, Comté et Bretagne (années 1440-1490)», p. 125-148.

32. É. LECUPPRE-DESJARDIN, Le Royaume inachevé des ducs de Bourgogne (XIVe-XVe siècles), Paris, 2016.

33. C. MotTIER, «Entre Savoie, Bourgogne et France : tradition de service multiple d'un lignage bressan, les Coucy-Châteauvieux ( $\mathrm{XV}^{\mathrm{e}}-\mathrm{XVI}^{\mathrm{e}}$ siècles) », p. 91-106.

34. P.-G. GIRAULT, «Les Carondelet: ascension sociale, commande artistique et construction lignagère entre Bresse, Franche-Comté et Pays-Bas (Xve-XviII ${ }^{\mathrm{e}}$ siècle) », p. 33-56.

35. J. LOISEAU, « La Couronne de France et la noblesse de Bourgogne (1595-1635) », p. 77-89.

36. "Autour des Chalon et de la noblesse en pays bourguignons ( $\mathrm{XIV}^{\mathrm{e}}-\mathrm{XVI}^{\mathrm{e}}$ siècle) », $58^{\mathrm{e}}$ Rencontres du Centre d'études bourguignonnes, Lons-le-Saunier et Arlay, 21-24 septembre 2017. 


\section{AUTEUR}

\section{FLORENTIN BRIFFAZ}

Doctorant contractuel en Histoire médiévale à l'Université Lumière-Lyon 2, CIHAM - UMR 5648 